\section{Variable outcome and methylation status according to CEBPA mutant type in double-mutated acute myeloid leukemia patients and the possible implications for treatment} Dima El-Sharkawi, ${ }^{1}$ Duncan Sproul, ${ }^{2}$ Christopher G. Allen, ${ }^{1}$ Andrew Feber, ${ }^{3}$
Melissa Wright, ${ }^{4}$ Robert K. Hills, ${ }^{4}$ David C. Linch ${ }^{1}$ and Rosemary E. Gale ${ }^{1}$

${ }^{1}$ Department of Haematology, UCL Cancer Institute, London; ${ }^{2} \mathrm{MRC}$ Human Genetics Unit and Edinburgh Cancer Research Centre, MRC Institute of Genetics and Molecular

Medicine, University of Edinburgh, Edinburgh; ${ }^{3}$ Medical Genomics, UCL Cancer Institute, London and ${ }^{4}$ Centre for Trials Research, Cardiff University, UK

\section{ABSTRACT}

A lthough CEBPA double-mutated (CEBPA $\left.{ }^{\mathrm{DM}}\right)$ acute myeloid leukemia is considered to be a favorable-risk disease, relapse remains a major cause of treatment failure. Most $C E B P A^{\mathrm{DM}}$ patients have a classic biallelic mutant combination with an $\mathrm{N}$-terminal mutation leading to production of p30 protein plus a C-terminal loss-offunction in-frame indel mutation (CEBPA ${ }^{\text {Classic-DM }}$ ), but approximately onethird of cases have one or more non-classic mutations, with diverse combinations reported, and there is little information on the consequences of such mutants. We evaluated outcome in a cohort of $104 C E B P A^{\mathrm{DM}}$ patients, 79 CEBPA $A^{\text {Classic-DM }}$ and 25 with non-classic mutants, and found that the latter may have poorer survival (5-year overall survival $64 \% v s$. $46 \% ; P=0.05)$, particularly post relapse ( $41 \%$ vs. $0 \% ; P=0.02)$. However, for this analysis, all non-classic cases were grouped together, irrespective of mutant combination. As CEBPA ${ }^{\mathrm{DM}}$ cases have been reported to be hypermethylated, we used methylation profiling to assess whether this could segregate the different mutants. We developed a CEBPAClassic-DM methylation signature from a preliminary cohort of $10 C E B P A^{\mathrm{DM}}$ (including $\left.8 C E B P A^{\text {Classic-DM }}\right)$ and $30 C E B P A$ wild-type $\left(C E B P A^{\mathrm{WT}}\right)$ samples, and independently validated the signature in $17 C E B P A^{\text {Classic-DM }}$ cases. Assessment of the signature in $16 C E B P A^{\mathrm{DM}}$ cases with different non-classic mutant combinations showed that only $31 \%$ had a methylation profile equivalent to $C E B P A^{\text {Classi-DM }}$ whereas for $69 \%$ the profile was either intermediate between $C E B P A^{\text {Classic-DM }}$ and $C E B P A^{\mathrm{WT}}$ or equivalent to $C E B$ $P A^{\mathrm{WT}}$. These results suggest that $C E B P A^{\mathrm{DM}}$ cases with non-classic mutants may be functionally different from those with $C E B P A^{\text {Classic-DM }}$ mutants, and should not automatically be included in the same prognostic group. (AML12 is registered under ISRCTN17833622 and AML15 under ISRCTN17161961).

\section{Introduction}

The CEBPA gene encodes CCAAT/enhancer binding protein- $\alpha$ (C/EBP $\alpha)$, a basic leucine zipper (bZIP) transcription factor that is essential for hematopoietic stem cell regulation and myeloid development. ${ }^{1,2}$ The gene is mutated in approximately $8 \%$ of acute myeloid leukemia (AML) patients with intermediate-risk cytogenetics, and presence of biallelic double mutations $\left(C E B P A^{\mathrm{DM}}\right)$ in the absence of an FLT3 internal tandem duplication $\left(F L T 3^{\text {ITD }}\right)$ is associated with a favorable prognosis ${ }^{3.9}$ In the current risk-adapted therapy strategies for AML, ${ }^{10-12}$ these patients are classified as good-risk and therefore not usually considered for consolidation of first remission by allogeneic transplantation. ${ }^{11,13}$

Mutations occur throughout the single exon gene but predominate at the $\mathrm{N}$ and $\mathrm{C}$ termini. ${ }^{14} \mathrm{~N}$-terminal mutations are nearly always frameshift or nonsense muta-
Haematologica 2018

Volume 103(1):91-100

\section{Correspondence: \\ rosemary.gale@ucl.ac.uk}

Received: May 19, 2017.

Accepted: October 10, 2017.

Pre-published: October 12, 2017.

doi:10.3324/haematol.2017.173096

Check the online version for the most updated information on this article, online supplements, and information on authorship \& disclosures: www.haematologica.org/content/103/1/91

\section{(C)2018 Ferrata Storti Foundation}

Material published in Haematologica is covered by copyright. All rights are reserved to the Ferrata Storti Foundation. Use of published material is allowed under the following terms and conditions:

https://creativecommons.org/licenses/by-nc/4.0/legalcode. Copies of published material are allowed for personal or internal use. Sharing published material for non-commercial purposes is subject to the following conditions:

https://creativecommons.org/licenses/by-nc/4.0/legalcode, sect. 3. Reproducing and sharing published material for commercial purposes is not allowed without permission in writing from the publisher. 
tions causing increased translation from an internal ATG start site and production of a truncated p30 protein that retains the same reading-frame as the full-length $\mathrm{p} 42$ protein but lacks the first transactivation domain (TAD1). The most common C-terminal mutations are in-frame indels in the bZIP DNA binding domain (DBD) or leucine zipper domain (LZD) that lead to loss of the ability to bind to DNA or dimerize, classified here as C-terminal loss-offunction (C-LOF). However, many other mutations have also been reported, including missense mutations in the $\mathrm{DBD}$ or LZD, missense mutations and in-frame indels in the mid-region, and frameshift or nonsense mutations in the mid-region or C-terminus. Some also present as homozygous alterations due to chromosome 19 uniparental disomy. ${ }^{15,16}$ The most common combination of mutations is an N-terminal frameshift on one allele plus a C-terminal in-frame indel on the other allele that together are predicted to lead to complete loss of normal p42 $\mathrm{C} / \mathrm{EBP} \alpha$ activity, ${ }^{14}$ hereafter called the classic CEBPA ${ }^{\mathrm{DM}}$ combination (CEBPA $\left.{ }^{\text {Classic-DM }}\right)$. This combination was identified in 204 of 305 CEBPA ${ }^{\text {DM }}$ cases $(67 \%)$ with defined mutants reported in six studies containing 20 or more CEBPA ${ }^{\mathrm{DM}}$ cases. ${ }^{3,6,8,17-19}$ The remaining 101 cases had multiple different mutant combinations with diverse consequences: 54 (18\% of total CEBPA $\left.{ }^{\mathrm{DM}}\right)$ would be predicted to produce just p30 due to the presence of a mid-region or Cterminal truncation, $12(4 \%)$ would only produce a classic C-LOF protein, 19 (6\%) p30 plus a C-terminal missense mutant, $5(2 \%)$ just a C-missense mutant, and $11(4 \%)$ other mutant combinations.

Understanding the consequence of the different types of CEBPA ${ }^{\mathrm{DM}}$ mutants is needed as this may impact on clinical outcome, but there is limited information available on the specific mutations. CEBPA ${ }^{\mathrm{DM}}$ cases have gene expression profiles that are significantly different from single-mutated $\left(C E B P A^{\mathrm{SM}}\right)$ and wild-type $\left(C E B P A^{\mathrm{WT}}\right)$ cases. ${ }^{3,7}$ However, recent data suggest that $C E B P A^{\mathrm{DM}}$ cases with non-classic combinations may not always cluster with other $C E B P A^{\mathrm{DM}}$ cases, ${ }^{7,20}$ with 3 of 7 such cases classified as negative for the $C E B P A^{\mathrm{DM}}$ expression profile in one report. ${ }^{20}$ Furthermore, genotype stratification according to expression profiling may be confounded by CEBPA ${ }^{\mathrm{WT}}$ cases with completely silenced CEBPA expression $\left(C E B P A^{\text {SII }}\right)$ due to methylation of the CEBPA promoter as these cases cluster together with $C E B P A^{\mathrm{DM}}$ cases. ${ }^{21} C E B P A^{\mathrm{DM}}$ cases also form distinct epigenetic clusters with a markedly hypermethylated profile. ${ }^{22,23}$ But although $C E B P A^{\text {SIL }}$ cases also have a hypermethylated profile, this segregates from the CEBPA ${ }^{\mathrm{DM}}$ cluster and, interestingly, they appear to be associated with a biologically distinct subtype of AML with a poor prognosis. ${ }^{21,22,24}$ Potential methylation differences according to the underlying combination of CEBPA mutants, however, have not been reported.

In order to investigate potential differences between $C E B P A^{\mathrm{DM}}$ mutant combinations, we evaluated clinical outcome in 104 CEBPA ${ }^{\mathrm{DM}}$ cases (79 CEBPA $A^{\text {Classic-DM }}$ and 25 with non-classic mutants) and observed that the non-classic cases may have a lower overall survival (OS). However, the number of cases was relatively small and all cases with a non-classic mutant were included in this group, irrespective of the mutant type. We therefore investigated whether methylation profiling of samples from doublemutated patients could assist in segregating the different mutant combinations.

\section{Methods}

\section{Patient cohorts}

The patients investigated were younger adults entered into the UK MRC AML10, AML12 and AML15 trials. Informed patient consent was obtained in accordance with the Declaration of Helsinki, and ethical approval for tissue use from the Wales Research Ethics Committee 3. Clinical outcome was evaluated in $104 C E B P A^{\mathrm{DM}}$ patients, all under 60 years of age, and methylation profiling was performed on 135 patients: 132 (98\%) of them under 60 years of age (Online Supplementary Figure S1). Selected tests were performed on a further 82 samples with specific cytogenetic and molecular abnormalities.

\section{Therapy, clinical end points and statistical methods}

Details of clinical protocols, end points and statistical methods are defined in the Online Supplementary Appendix.

\section{Methylation arrays and data processing}

DNA was bisulfite-converted using the EZ DNA MethylationGold Kit (Zymo Research, California, USA) and random samples checked by methylation-specific PCR to ensure efficient conversion (see the Online Supplementary Appendix). Methylation profiling was performed using the Illumina Infinium HumanMethylation27 ( $\mathrm{n}=40, \quad$ cohort 1$) \quad$ and HumanMethylation450 ( $\mathrm{n}=95$, cohort 2) BeadChip arrays (Illumina Inc., California, USA). Details of data processing are given in the Online Supplementary Appendix. Derived $\beta$ values were expressed as the percentage methylation at a given $\mathrm{CpG}$ probe. Selected CpG sites were further analyzed using pyrosequencing assays (see the Online Supplementary Appendix).

\section{Unsupervised analysis of patient methylation profiles}

In a given sample, probes were defined as methylated if the $\beta$ value was more than 0.3 , unmethylated if 0.3 or under. ${ }^{25}$ Samples were clustered based on their $\beta$ values at probes displaying significant variation (variable probes), as previously defined. ${ }^{25,26}$ They were defined as variable if methylated in 1 sample or more plus unmethylated in 1 sample or more. CpG islands (CGIs) were located as previously defined; ${ }^{27}$ their methylation levels were derived by calculating the mean $\beta$ value of probes at these locations. Hierarchical clustering was performed using the Euclidian distance of $\beta$ values and Ward algorithm in $R$.

\section{Derivation and analysis of CEBPA signature}

A methylation signature of CEBPA genotype was derived from variably methylated $\mathrm{CpGs}$ in cohort 1 . Signature CpGs were selected as the top 25 ranked probes based on the mean rank of $P$-values of Wilcoxon tests and the absolute median difference in $\beta$ value between $C E B P A^{\text {Classi-DM }}$ and $C E B P A^{\mathrm{WT}}$ samples. Probes were included in the analysis if more than $90 \%$ of samples and 2 or more CEBPA $A^{\text {Classic-DM }}$ and 2 or more $C E B P A^{\mathrm{WT}}$ samples had observable data. Methylation signatures of CEBPA $A^{\text {Classic-DM }}$ and CEBPA ${ }^{\mathrm{WI}}$ samples were then defined as the median $\beta$ values observed at these 25 probes. Samples were scored relative to these signatures by calculating the Euclidian distance between the signatures and their profiles at signature probes.

\section{Mutant CEBPA level and confirmation of biallelic status}

Mutant CEBPA level was quantified as previously described ${ }^{6}$ or approximated from the sequence chromatogram (average height of $\geq 5$ peaks). Monoallelic/biallelic status was investigated by sequencing clones derived from full-length CEBPA amplicons as previously described. ${ }^{6}$ 
Table 1. CEBPA genotype of investigated cohorts.

\begin{tabular}{|c|c|c|c|}
\hline & CEBPA genotype* & Number & Predicted functional consequence \\
\hline \multirow{11}{*}{$\begin{array}{l}\text { Clinical cohort } \\
(n=104)\end{array}$} & $C E B P A^{\text {Classic-DM }}$ & 79 & $\mathrm{p} 30+\mathrm{C}-\mathrm{LOF}$ \\
\hline & \multicolumn{3}{|l|}{ Non-classic CEBPA ${ }^{\mathrm{DM}}$} \\
\hline & Classic N + C-missense & 4 & $\mathrm{p} 30+\mathrm{C}-\mathrm{LOF}$ \\
\hline & Classic N + mid-frameshift & 5 & p30 \\
\hline & Classic N + C-frameshift & 2 & p30 \\
\hline & Homozygous classic $\mathrm{N}$ & 1 & p30 \\
\hline & Homozygous classic $\mathrm{C}$ & 4 & C-LOF \\
\hline & Homozygous C-missense & 3 & C-LOF \\
\hline & Classic C + C-frameshift & 4 & C-LOF \\
\hline & Classic C + C-missense & 1 & C-LOF \\
\hline & Mid-frameshift + C-missense & 1 & C-LOF \\
\hline Methylation & $C E B P A^{\text {Classic-DM }}$ & 8 & $\mathrm{p} 30+\mathrm{C}-\mathrm{LOF}$ \\
\hline \multirow[t]{4}{*}{ Cohort $1(n=40)$} & \multicolumn{3}{|l|}{ Non-classic $C E B P A^{\mathrm{DM}}$} \\
\hline & Classic C + C-frameshift & 1 & C-LOF \\
\hline & Homozygous C-missense & 1 & C-LOF \\
\hline & $C E B P A^{\mathrm{WT}}$ & 30 & WT \\
\hline Methylation & $C E B P A^{\text {Classic-DM }}$ & 17 & $\mathrm{p} 30+\mathrm{C}-\mathrm{LOF}$ \\
\hline \multirow[t]{16}{*}{ Cohort $2(\mathrm{n}=95)$} & \multicolumn{3}{|l|}{ Non-classic CEBPA $A^{\mathrm{DM}}$} \\
\hline & Classic N + C-missense & 3 & $\mathrm{p} 30+\mathrm{C}-\mathrm{LOF}$ \\
\hline & Classic N + mid-frameshift & 5 & p30 \\
\hline & Classic N + C-frameshift & 1 & p30 \\
\hline & Homozygous classic C & 2 & C-LOF \\
\hline & Homozygous C-missense & 2 & C-LOF \\
\hline & Classic C + C-missense & 1 & C-LOF \\
\hline & \multicolumn{3}{|l|}{$C E B P A^{\mathrm{sM}}$} \\
\hline & Classic N & 9 & $\mathrm{p} 30+\mathrm{WT}$ \\
\hline & Classic C & 5 & C-LOF + WT \\
\hline & Mid-indel & 3 & UNK + WT \\
\hline & Mid-frameshift & 9 & Null$^{* *}+$ WT \\
\hline & Mid-missense & 5 & UNK + WT \\
\hline & C-frameshift & 2 & Null** + WT \\
\hline & C-missense & 5 & $\mathrm{C}-\mathrm{LOF}+\mathrm{WT}$ \\
\hline & $C E B P A^{\mathrm{WT}}$ & 26 & WT \\
\hline
\end{tabular}

*Details of the specific mutations are given in Online Supplementary Table S1. * Mid-region or C-terminal mutants with a truncating frameshift or nonsense mutation. C: C-terminal mutation; C-LOF: C-terminal loss-of-function; DM: double mutant; indel: in-frame insertion and/or deletion; N: N-terminal mutation; SM: single mutant; UNK: unknown; WT: wild-type.

\section{Results}

\section{Clinical outcome in CEBPA ${ }^{\mathrm{DM}}$ cases according to mutant type}

Of the $104 C E B P A^{\text {DM }}$ cases evaluated, 79 had classic and 25 had non-classic mutants (Table 1 and Online Supplementary Table S1). The latter included 4 patients predicted to produce p30 plus a C-terminal missense mutant, 8 with combinations predicted to produce just $\mathrm{p} 30$, and 13 with different combinations predicted to produce just a CLOF protein. There was no difference in the baseline characteristics between the classic and non-classic cases, including white cell count, sex, World Health Organization (WHO) performance status, and AML type (de novo or secondary), although the non-classic cases were older (median 35 vs. 47 years; $P=0.001$ ) (Online Supplementary Table S2). All classic cases had intermediaterisk cytogenetics compared to $89 \%$ of the non-classic cases $(P=0.05)$. There was no significant difference in the incidence of FLT3 ${ }^{\mathrm{ID}}$, NPM1, IDH1, WT1, TET2 and GATA2 mutations between the groups, although the non-classic cases had more IDH2 mutations ( $1 \%$ vs. $20 \%, P=0.003$; all $I D H 2^{\mathrm{R} 1400}$ ) and DNMT3A mutations ( $3 \%$ vs. $16 \%, P=0.03$ ). Median follow up was 9.5 years (range: $0.1-22$ years). Neither the proportion of transplanted patients, the type of transplant, nor the stage of transplantation differed between the groups (Online Supplementary Table S2).

$C E B P A^{\text {Classic-DM }}$ cases had a slightly higher but statistically non-significantly different complete remission (CR) rate to non-classic cases ( $95 \%$ vs. $88 \%, P=0.2)$ (Table 2 ). In uni- 
A

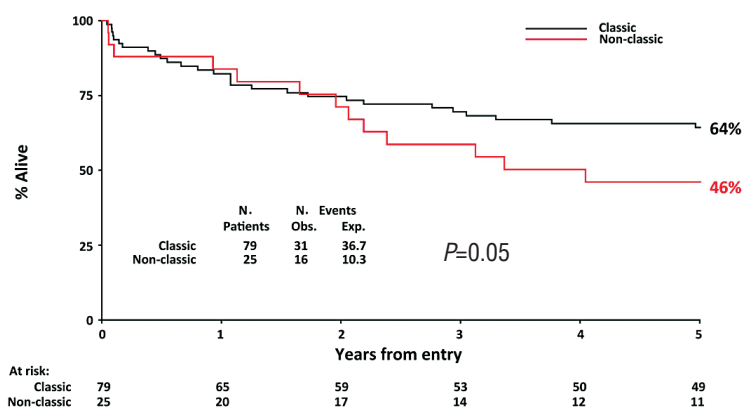

C

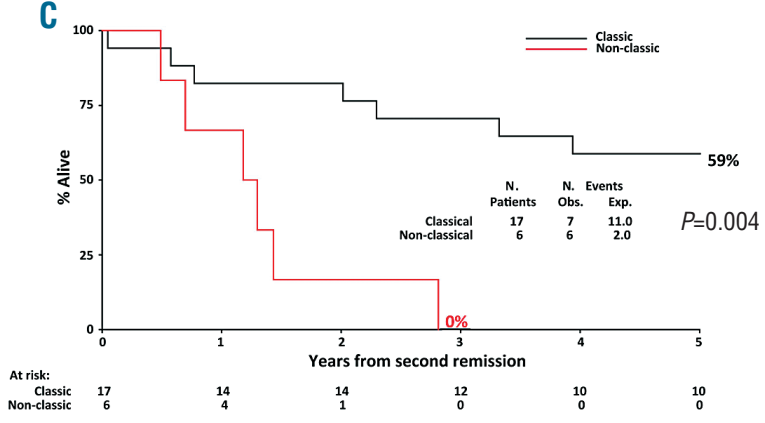

B

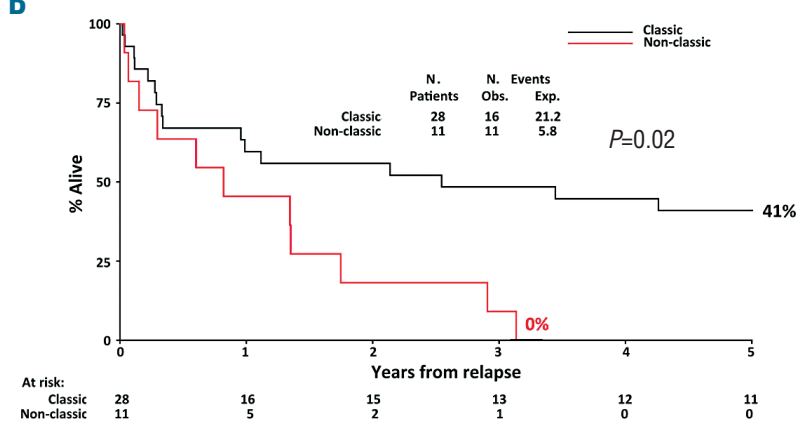

D

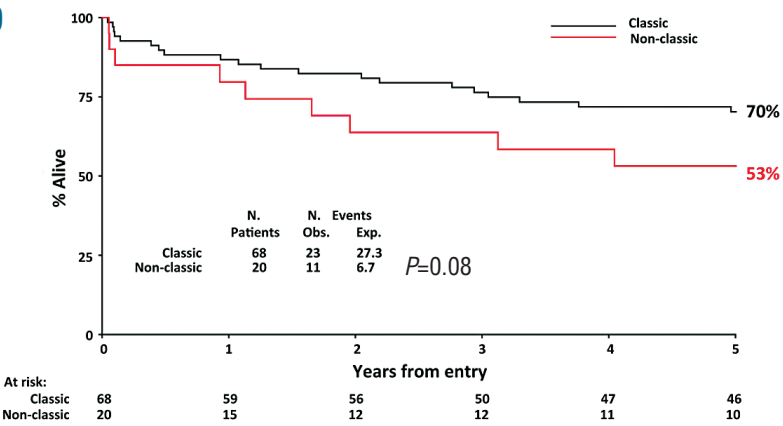

Figure 1.Clinical outcome in CEBPA ${ }^{\mathrm{DM}}$ patients according to mutant combination. (A) Overall survival in the total cohort of 104 CEBPA ${ }^{\mathrm{DM}}$ patients. (B) Survival post relapse in the 39 patients who relapsed. (C) Survival from second remission in the 23 relapsed patients who achieved a second remission. (D) Overall survival in the total cohort excluding patients with FLT3 ${ }^{\text {ITD }}$ and DNMT3A mutations.

variate analysis, there was no significant difference in relapse-free survival or relapse rate (RR). However, there was a trend towards a lower OS in the non-classic cases (64\% vs. $46 \%$ at 5 years, $P=0.05$ ) (Figure $1 \mathrm{~A}$ ), which was largely due to the worse outcome post-relapse in the nonclassic cases. The proportion of relapsing patients who achieved a second remission did not differ between the groups $(61 \%$ vs. $55 \%)$, but 5 -year survival post relapse was $41 \%$ versus $0 \%(P=0.02)$ (Figure $1 \mathrm{~B})$, and $59 \%$ versus $0 \%$ from second remission $(P=0.004)$ (Figure $1 \mathrm{C})$. The survival differences were not, however, statistically different in multivariate analysis (Table 2), but this is not surprising given the small group sizes. Although the significantly higher proportion of DNMT3A-mutated cases in the nonclassic group could have contributed to their worse outcome, as both these mutations and $F L T 3^{\mathrm{IDD}}$ adversely impact on the favorable outcome associated with CEBPA ${ }^{\mathrm{DM}} \mathrm{AML}^{6,28}$ the trend towards a lower OS in the non-classic cases was still present when patients with these mutations were excluded $(70 \%$ vs. 53\%; Hazard Ratio 1.95, 95\% confidence intervals $0.95-4.01 ; P=0.08$ ) (Figure 1D).

\section{Development of a CEBPA ${ }^{\text {classic-DM }}$ methylation signature}

The clinical evaluation grouped all non-classic cases together in order to obtain an adequate number of patients for analysis, but this cohort, therefore, included patients with many mutant combinations predicted to have differing functional consequences. In order to explore potential methods for discriminating between these combinations, we investigated the impact of mutant type on methylation profiles. A preliminary cohort of samples from 40 normal karyotype (NK) FLT3 ${ }^{\mathrm{WT}} N P M 1^{\mathrm{WT}}$ patients were investigated using the Illumina Infinium HumanMethylation27 array: 10 were $C E B P A^{\mathrm{DM}}$ and 30 were $C E B P A^{\mathrm{WT}}$ (Methylation cohort 1) (Table 1), approximating the relative proportions of such cases in NK FLT3 ${ }^{\mathrm{WT}} N P M 1^{\mathrm{WT}}$ patients in our earlier study. ${ }^{6}$ The array data were validated by pyrosequencing assays at four differentially methylated CpG sites (Online Supplementary Figure S2). Most CpG sites analyzed showed little variation in methylation levels across the whole cohort, but unsupervised cluster analysis according to levels of the most variably methylated probes revealed two main clusters. All $10 C E B P A^{\mathrm{DM}}$ samples, including two non-classic cases, fell in the cluster of 16 samples with significantly higher levels of mean CGI methylation (Figure 2A), and the mean level of CGI methylation was significantly different between $C E B P A^{\text {Classic-DM }}$ and $C E B P A^{\text {WT }}$ samples (Figure 2B).

A supervised approach was then used to create CEBPA $A^{\text {Classic-DM }}$ and CEBPA ${ }^{\mathrm{WT}}$ methylation signatures based on the 25 most differentially methylated sites between the $C E B P A^{\text {Classic-DM }}$ and CEBPA ${ }^{\mathrm{WT}}$ samples (Figure $2 \mathrm{C}$ and Online Supplementary Table S3). Two distance scores were calculated for each sample based on the Euclidian distance between their methylation levels at these signature probes and the median profile of the CEBPA $A^{\text {Classic-DM }}$ and CEBPA ${ }^{\mathrm{WI}}$ samples. This confirmed that, when assessed according to 
Table 2. Outcome according to CEBPA ${ }^{\mathrm{DM}}$ mutant combination.

\begin{tabular}{|c|c|c|c|c|}
\hline Outcome & $\begin{array}{l}\text { Classic } \\
\text { CEBPA }^{\mathrm{DM}} \\
(\mathrm{n}=79)\end{array}$ & $\begin{array}{c}\text { Non-classic } \\
\text { CEBPADM } \\
(n=25)\end{array}$ & $\begin{array}{c}\text { CEBPAA }{ }^{\text {classic.DM }} \text { Vs. no } \\
\text { OR or HR } \\
\text { Univariate }\end{array}$ & $\begin{array}{l}\text { sic CEBPA }{ }^{\text {sm }} \\
\text { CI), } P \\
\quad \text { Multivariate* }\end{array}$ \\
\hline $\mathrm{CR}$ & $95 \%$ & $88 \% * *$ & $3.20(0.53-19.47), P=0.2$ & Not evaluable*** \\
\hline 5 -year OS & $64 \%$ & $46 \%$ & $1.84(1.01-3.37), P=0.05$ & $1.44(0.74-2.79), P=0.3$ \\
\hline 5-year RFS & $49 \%$ & $45 \%$ & $1.15(0.61-2.16), P=0.7$ & $1.03(0.52-2.06), P=0.9$ \\
\hline 5-year RR & $41 \%$ & $47 \%$ & $1.32(0.66-2.66), P=0.4$ & $1.09(0.47-2.55), P=0.8$ \\
\hline
\end{tabular}

*Adjusted for age, white blood cell count,World Health Organization performance status, type of leukemia, sex,FLT3 and DNMT3A genotype. **Remission status was missing for one patient. ${ }^{* *}$ Insufficient events for analysis. $P$ : $P$-value; n: number; Cl: confidence intervals; CR: complete remission; DM: double mutant; HR: hazard ratio; OR: odds ratio OS: overall survival; RFS: relapse-free survival; RR: relapse rate.

their distance scores, the CEBPA ${ }^{\text {Classic-DM }}$ samples formed a distinct group that were clearly separate from the $C E B P A^{\mathrm{WT}}$ samples (Figure $3 \mathrm{~A}$ ).

The $C E B P A^{\text {Classic-DM }}$ methylation signature was validated with samples from a further $17 C E B P A^{\text {Classic-DM }}$ and 26 $C E B P A^{\mathrm{WT}}$ cases (Methylation cohort 2) (Table 1) using the HumanMethylation 450 array. Sixteen of the 17 CEBPA ${ }^{\text {Classic-DM }}$ cases $(94 \%)$ fell in the same cluster in unsupervised analysis, with a relatively more hypermethylated profile, and all CEBPA ${ }^{\mathrm{WT}}$ cases fell in the hypomethylated cluster (Online Supplementary Figure S3). Using supervised analysis according to the derived CEBPA Classic-DM and $C E B P A^{\mathrm{WT}}$ signatures, the same $16 C E B P A^{\mathrm{DM}}$ cases had a methylation profile that was closest to the $C E B P A^{\text {Classic-DM }}$ signature with a wide difference between the two signatures (Figures $3 \mathrm{~B}$ and 4 ), indicating that their profile was equivalent to the CEBPA ${ }^{\text {Classi-DM }}$ cases in cohort 1 . Further analysis of the one CEBPA ${ }^{\text {Classic-DM }}$ case that fell closer to the $C E B P A^{\mathrm{WT}}$ group indicated that the mutations were biallelic but only approximately half of the cells in the sample carried mutations; mean mutant level was $28 \%$ for the pair, which was the lowest mean level of all 25 CEBPA ${ }^{\text {Classic-DM }}$ cases (median $44 \%$; range: $28-50 \%$ ). The methylation profile of this case could, therefore, have been affected by the presence of a significant proportion of non-leukemic cells and it was excluded from further analyses. Using the distance scores (mean $\pm 2 \mathrm{SD}$ ) for $C E B P A^{\text {Classic-DM }}$ cases in cohort 1 , tests showed that these scores classified CEBPA ${ }^{\text {Classic-DM }}$ and CEBPA ${ }^{\mathrm{WT}}$ genotypes in the second cohort with $95 \%$ accuracy, $88 \%$ sensitivity, and $100 \%$ specificity. The distance scores of the 24 $C E B P A^{\text {Classic-DM }}$ cases from the combined cohorts were then used to define a $C E B P A^{\text {Classic-DM }}$ quadrant that segregated all $C E B P A^{\text {Classic-DM }}$ cases from CEBPA ${ }^{\mathrm{WT}}$ cases (Figure $3 \mathrm{C}$ ).

\section{Investigation of the CEBPA ${ }^{\text {classic-DM }}$ methylation signature in other good-risk patients}

In order to examine whether the CEBPA ${ }^{\text {Classic-DM }}$ methylation signature could simply reflect 'good-risk' disease or be due to a lack of C/EBP $\alpha$ expression, methylation levels at three differentially methylated $\mathrm{CpG}$ sites from the signature were quantified by pyrosequencing using samples from 21 patients with inv(16) and 19 with t(8;21), both associated with down-regulated C/EBP $\alpha$ expression, ${ }^{29-31}$ and 42 with NPM1 ${ }^{\mathrm{MUT}}$ FLT $3^{\mathrm{WT}}$. KHNYN and VAMP5 were more hypermethylated in the CEBPA $A^{\text {Classic-DM }}$ signature, while $L Y 9$ was more hypomethylated. Each subgroup differed significantly from the $24 C E B P A^{\text {Classic-DM }}$ cases at two of the three sites (Online Supplementary Figure S4). A com- posite methylation score was calculated for each sample; it was statistically significantly different from the $C E B P A^{\text {Classic-DM }}$ cases for all three subgroups (Figure 5), indicating that the methylation profile was a distinct feature of the mutant proteins that lead to total loss of normal $\mathrm{C} / \mathrm{EBP} \alpha$ function rather than absence of $\mathrm{C} / \mathrm{EBP} \alpha$ per se.

\section{Investigation of non-classic CEBPA ${ }^{\mathrm{DM}}$ and CEBPA ${ }^{\mathrm{SM}}$ mutants}

Having established that $C E B P A^{\text {Classic-DM }}$ cases have a methylation profile that is distinct from $C E B P A^{\mathrm{WT}}$ cases, profiles of $14 C E B P A^{\mathrm{DM}}$ cases with a variety of different non-classic combinations were investigated using the HumanMethylation450 array (Methylation cohort 2) (Table 1). On unsupervised analysis, 9 (64\%) were hypermethylated and 5 (36\%) hypomethylated (Online Supplementary Figure S3). To assess their impact on the CEBPA methylation signatures, these 14 cases and the 2 non-classic cases from the initial cohort were considered according to the predicted functional consequence of the combination.

Six cases were predicted to produce just p30 protein, with a classic $\mathrm{N}$ plus null mutant (mid-region or C-terminal frameshift/nonsense) combination. Only 1 fulfilled the $C E B P A^{\text {Classic-DM }}$ criteria; 4 fell outside this quadrant but were still distinct from $C E B P A^{\mathrm{WT}}$ cases, and 1 grouped with the $C E B P A^{\mathrm{WT}}$ cases (Figure 6A). Three cases were predicted to just give rise to classic C-LOF proteins without p30 (2 homozygous classic C, 1 compound heterozygous with a classic C plus C-frameshift combination). Two fulfilled the $C E B P A^{\text {Classic-DM }}$ criteria and 1 was intermediate between $C E B P A^{\text {Classic-DM }}$ and CEBPA ${ }^{\mathrm{WT}}$. The 7 cases with missense mutations were also highly variable. Three had a classic $N$ plus C-missense combination; none fulfilled the $C E B P A^{\text {Classic-DM }}$ criteria, 2 were intermediate between the signatures, and 1 grouped with $C E B P A^{\mathrm{WT}}$ cases. Mutant levels were indicative of $80 \%$ or more mutated cells in all 3 cases; 2 were biallelic by cloning but no full-length amplicons could be obtained in 1 case. Similarly, only 1 of the 3 homozygous C-missense cases fell in the $C E B P A^{\text {Classic-DM }}$ quadrant, the other 2 were equivalent to $C E B P A^{\mathrm{WT}}$ cases. The remaining case was compound heterozygous with a classic $C$ and C-missense combination, and this did fulfil the CEBPA $A^{\text {Classic-DM }}$ criteria.

These results suggest that the functional consequence of double-mutated cases producing at least 1 non-classic mutant protein can be highly variable and difficult to predict. Of note, when outcome was assessed in the non-classic cases according to their methylation profile, there was 
a suggestion that those that fulfilled the CEBPA Classic-DM methylation criteria were less likely to relapse, with only 1 of 4 cases $(25 \%)$ relapsing compared to 7 of 10 cases $(70 \%)$ that fell outside the CEBPA ${ }^{\text {Classic-DM }}$ quadrant (5-year RR $25 \%$ vs. $54 \%$, respectively). These numbers were too small for meaningful statistical analysis, and the results do not necessarily indicate a causal link between the methylation pattern and outcome. They do suggest, however, that the methylation pattern could be a useful biomarker, and thereby act as a surrogate for response and selection of therapy.

Methylation cohort 2 also included $38 C E B P A^{\mathrm{SM}}$ cases with a wide range of classic and non-classic mutants (Table 1). On unsupervised analysis, 31 (82\%) were in the hypomethylated cluster, with no apparent segregation between the $C E B P A^{\mathrm{SM}}$ and $C E B P A^{\mathrm{WT}}$ cases (Online

A
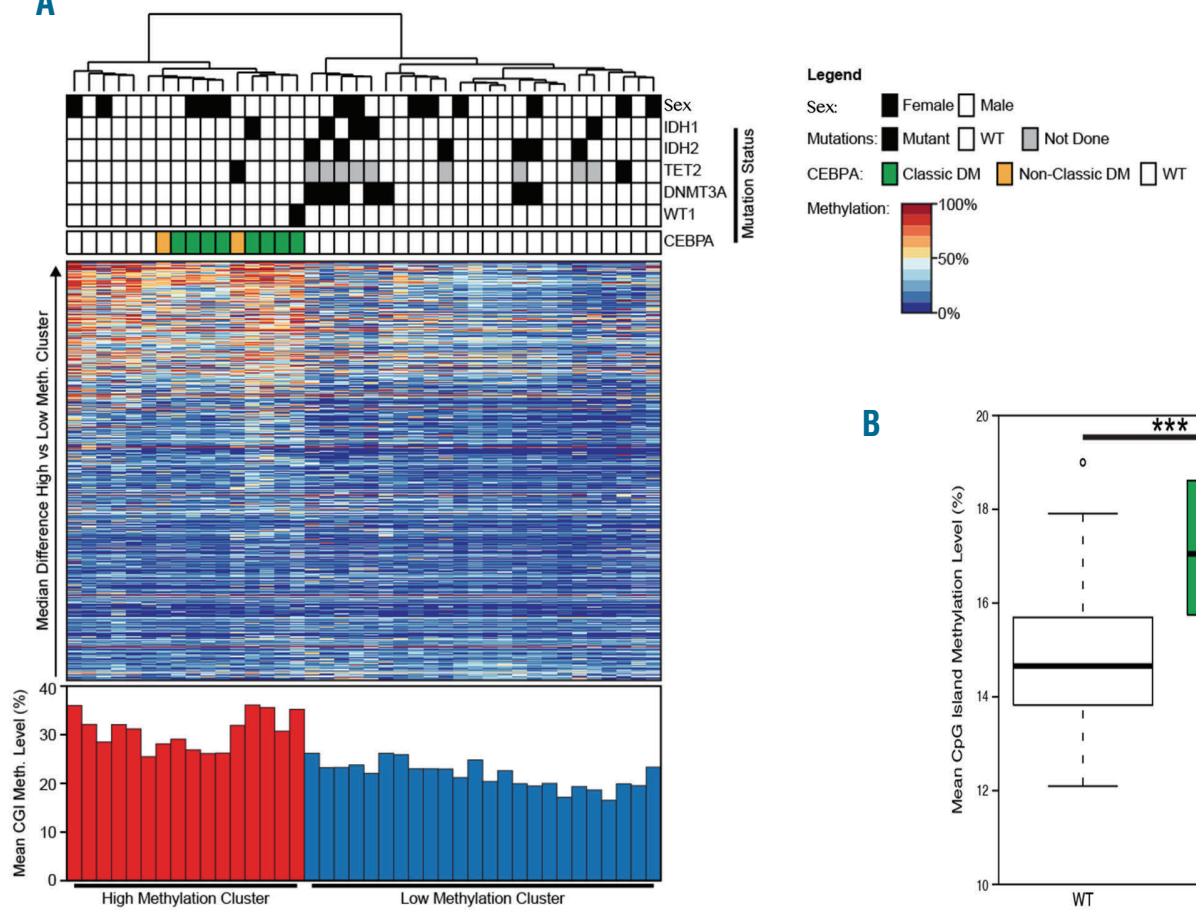

B
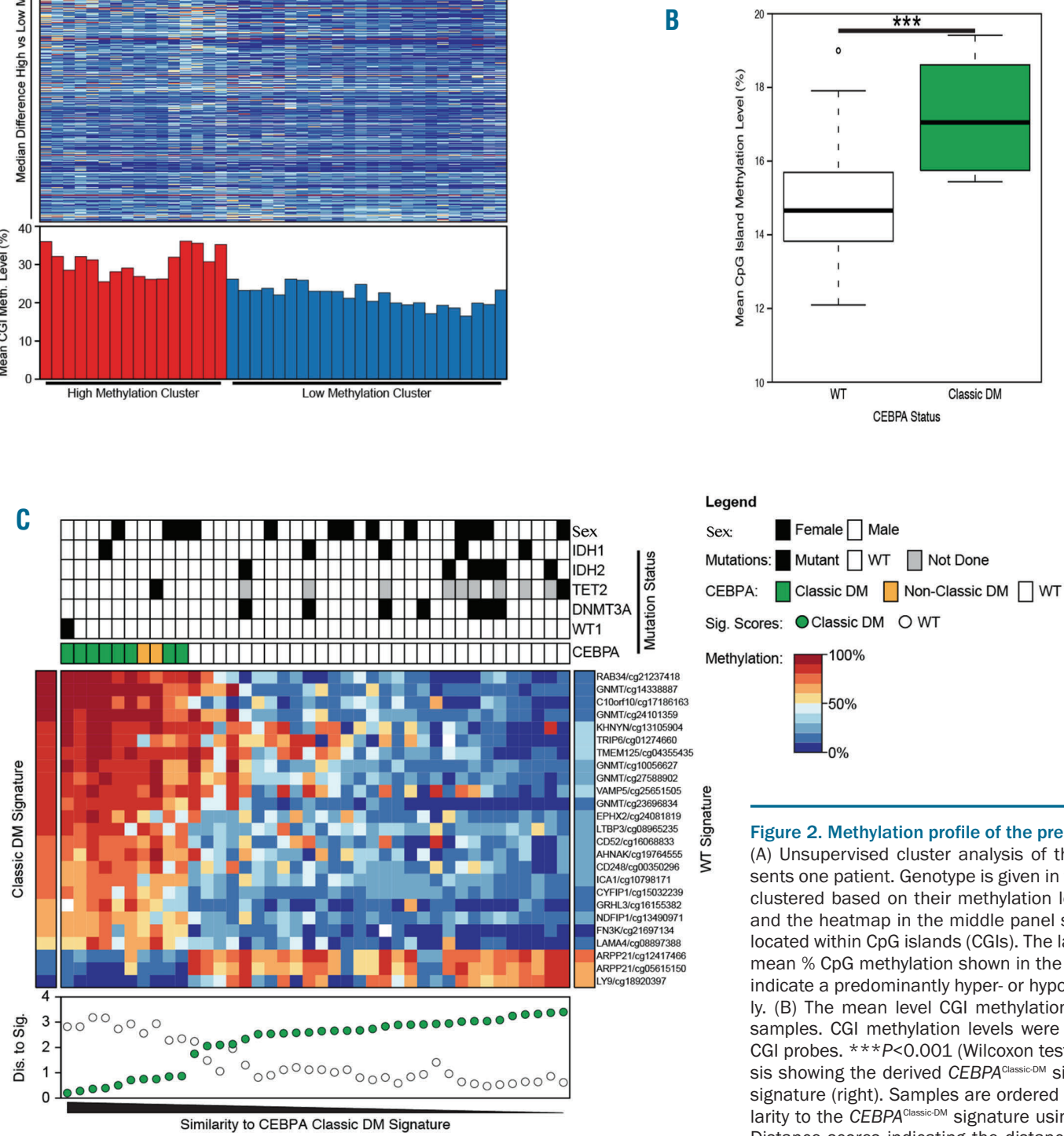

Figure 2. Methylation profile of the preliminary cohort of 40 samples. (A) Unsupervised cluster analysis of the cohort. Each column represents one patient. Genotype is given in the upper panel. Samples were clustered based on their methylation levels at 7679 variable probes, and the heatmap in the middle panel shows the variable $\mathrm{CpG}$ probes located within $\mathrm{CpG}$ islands (CGIs). The latter were used to calculate the mean \% CpG methylation shown in the lower panel; red and blue bars indicate a predominantly hyper- or hypo-methylated profile, respectively. (B) The mean level CGI methylation for $C E B P A^{\text {wT }}$ and $C E B P A^{\text {classic-DM }}$ samples. CGI methylation levels were calculated from all autosomal CGI probes. $* * * P<0.001$ (Wilcoxon test). (C) Supervised cluster analysis showing the derived CEBPA ${ }^{\text {Classi-DM }}$ signature (left) and the CEBPA ${ }^{\text {w }}$ signature (right). Samples are ordered according to their level of similarity to the CEBPA ${ }^{\text {classic-DM }}$ signature using Euclidian distance. (Bottom) Distance scores indicating the distance from the CEBPA ${ }^{\text {Classi-DM }}$ (green circles) and CEBPA ${ }^{\mathrm{wT}}$ (white circles) signatures; the lower the $y$-axis value, the more closely the sample matches that particular signature. 
Supplementary Figure S3). On supervised analysis, all except 2 cases were equivalent to CEBPA ${ }^{\mathrm{WT}}$ cases, with no obvious grouping according to the type of mutant (Figures 4 and $6 \mathrm{~B}$ ). The remaining 2 cases fell in or close to the $C E B P A^{\text {Classic-DM }}$ quadrant; both had a classic $C$ mutation. It is possible that the CEBPA ${ }^{\mathrm{WT}}$ allele had been silenced in these cases, but RNA samples were not available to check this.

\section{Discussion}

Molecular genotyping is increasingly used to risk stratify patients with AML, but clinical application of this information needs to be accurate and robust for optimal patient therapy. This is particularly important for good-risk patients such as those with biallelic CEBPA mutations, where the current recommendation is not to proceed to transplantation in first remission, as for some patients this could lead to undertreatment. Identifying those who are at greater risk of relapse and poorer survival may, therefore, guide patient management. Very limited information is available on the impact of the different CEBPA mutations, with all $C E B P A^{\mathrm{DM}}$ cases currently being classified as goodrisk, irrespective of the underlying mutant. However, mutations identified in approximately one-third of $C E B$ $P A^{\mathrm{DM}}$ patients do not conform to the classic combination of $\mathrm{N}$-terminal frameshift or nonsense mutation plus C-terminal in-frame indel. As many of the other mutations are unique and of unknown functional consequence, determining their significance is challenging, particularly in view of the multiple functions attributed to $\mathrm{C} / \mathrm{EBP} \alpha .{ }^{1,2}$

Although we had access to a database of 2162 patients with known CEBPA genotype and available clinical data from three consecutive UK MRC trials of younger adult patients with AML, $67 \%$ with intermediate-risk cytogenetics, we were still able to evaluate long-term outcome in a cohort of only $79 C E B P A^{\text {Classic-DM }}$ cases and $25 C E B P A^{\text {DM }}$ cases with at least one non-classic mutant. The results suggested that the non-classic cases may have a poorer outcome; in particular, none of these cases survived after relapse. These results may be influenced by differences in the coincident mutations in the two groups. The majority of recurrent mutations with known prognostic significance in AML are uncommon in CEBPA ${ }^{\mathrm{DM}}$ cases, ${ }^{32}$ and for many of them their impact in this subgroup is, therefore, not well defined. Both FLT3 ${ }^{\text {ID }}$ and DNMT3A mutations adversely impact on the favorable outcome of CEBPA ${ }^{\mathrm{DM}}$ AML. ${ }^{6,28}$ But even when patients with these mutations were excluded from the analysis, OS for the non-classic cases was still worse. The incidence of GATA2 mutations was non-significantly lower in the non-classic cases, but although one study reported that they are associated with better OS, ${ }^{33}$ most studies, including our own, observed no difference. ${ }^{34,35}$ There was a non-significantly higher incidence of TET2 mutations in the non-classic cases, but their impact is unclear, with only one study reported specifically for CEBPA ${ }^{\mathrm{DM}}$ AML that showed a worse OS but not event-free survival in TET2 ${ }^{\mathrm{MUT}}$ cases. $^{33}$ Clearly, many more cases would need to be analyzed in order to take into account coincident mutations other than FLT3 $3^{\text {IT }}$ and DNMT3A mutations.

A wide range of non-classic mutations was observed and, for the outcome analysis, sufficient patient numbers could only be obtained by grouping all the non-classic patients together, which precluded evaluation of specific mutant combinations. We, therefore, sought alternative methods of assessment and, as CEBPA ${ }^{\mathrm{DM}}$ patients have a distinct hypermethylated profile, ${ }^{22,23}$ investigated whether genome-wide methylation profiling could provide information on the more broad-spectrum functional consequence of different mutants. We confirmed the relatively hypermethylated profile associated with a CEBPA ${ }^{\mathrm{DM}}$ geno-

A

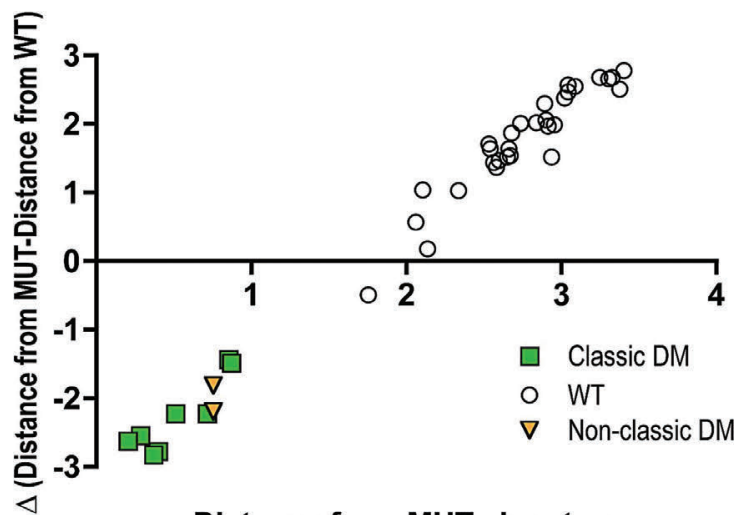

Distance from MUT signature

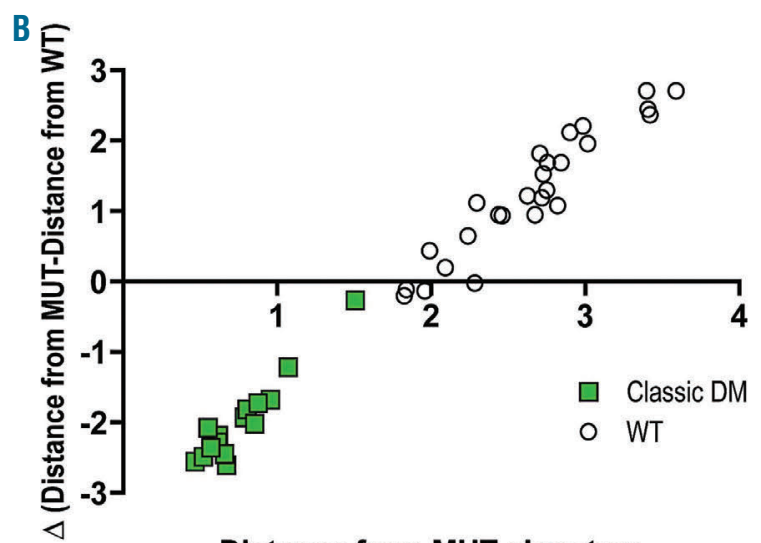

Distance from MUT signature

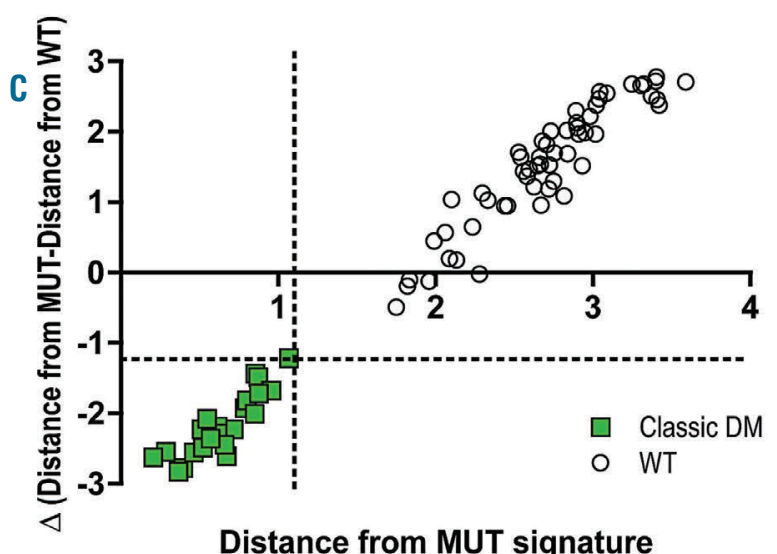

Figure 3. Analysis of the methylation profiles of the samples according to their distances from the derived CEBPA ${ }^{\text {Classic-DM }}$ and CEBPA ${ }^{\text {WT }}$ methylation signatures. Difference between the distance scores (CEBPA ${ }^{\text {Classic-DM }}$ - CEBPA ${ }^{\text {WT }}$ ) compared with the distance from the CEBPA ${ }^{\text {Classic.DM }}(\mathrm{MUT}$ ) signature of $(\mathrm{A})$ the preliminary cohort and (B) the CEBPA $A^{\text {Classic-DM }}$ and $C E B P A^{\text {wT }}$ cases in the follow-up cohort. (C) Criteria derived for CEBPA ${ }^{\text {Classic-DM }}$ using mean \pm 2 Standard Deviation (SD) of the distance scores of all 24 CEBPA ${ }^{\text {Classic-DM }}$ cases. 


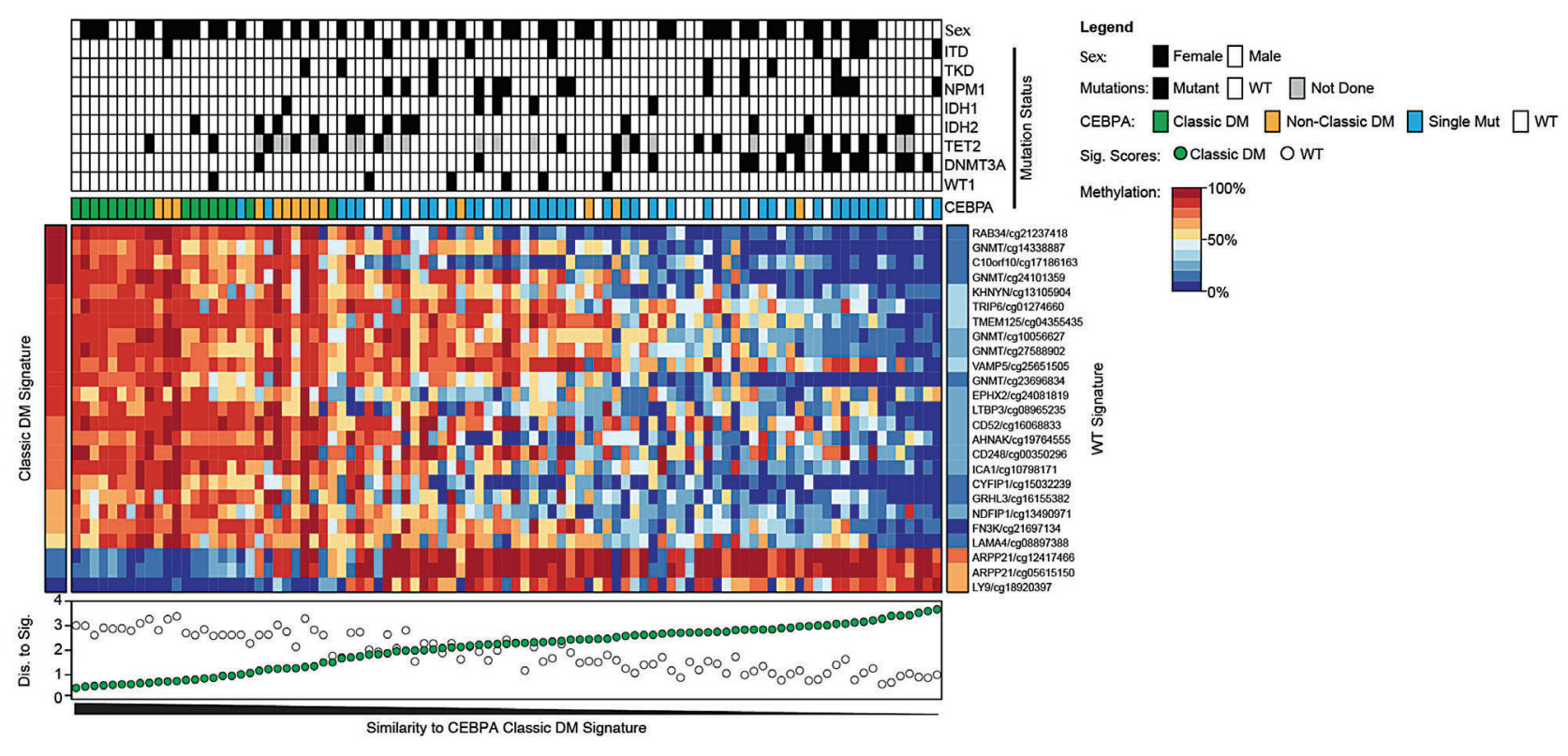

Figure 4. Analysis of the follow-up cohort of 95 samples. Supervised analysis according to the CEBPA classi-DM and CEBPA ${ }^{\text {wT }}$ methylation signatures. Patient CEBPA genotype is given above the heatmap. (Bottom) Distance scores indicating the distance from the CEBPA ${ }^{\text {classicDM }}$ (green circles) and CEBPA ${ }^{\text {wT }}$ (white circles) signatures; the lower the $y$-axis value, the more closely the sample matches that particular signature.

type in a preliminary cohort and then derived methylation signatures for $C E B P A^{\text {Classic-DM }}$ and $C E B P A^{\mathrm{WT}}$ cases using the 25 most differentially methylated $\mathrm{CpG}$ sites. These were validated in an independent cohort of samples, with 16 of the $17 C E B P A^{\text {Classic-DM }}$ cases studied clearly separating from the $C E B P A^{\mathrm{wT}}$ cases. The remaining case contained a significant proportion of non-mutated cells, which provided indirect evidence that the signatures reflected CEBPA genotype. The signatures were a distinct feature of mutant $\mathrm{C} / \mathrm{EBP} \alpha$ and could not simply be attributed to a lack of $\mathrm{C} / \mathrm{EBP} \alpha$, as they were not replicated in samples from patients with core-binding factor leukemias that are associated with downregulation of CEBPA expression. Presence of at least a minimal level of $\mathrm{C} / \mathrm{EBP} \alpha$ activity is thought to be necessary for the development of leukemia as Cebpa ${ }^{-1-}$ mice with totally absent $\mathrm{C} / \mathrm{EBP} \alpha$ accumulate immature myeloid progenitors but do not develop AML, ${ }^{36,37}$ and AML patients have not been reported with mutations leading to complete absence of C/EBP $\alpha$. These results, therefore, suggest that it is the functionally aberrant $\mathrm{C} / \mathrm{EBP} \alpha$ protein that underlies the hypermethylated profile detected in the CEBPA ${ }^{\mathrm{DM}}$ cases.

The $C E B P A^{\text {Classic-DM }}$ cases provided a framework for assessing the methylation profiles of mutant combinations with at least one non-classic mutant. Only $31 \%$ had a methylation profile equivalent to $C E B P A^{\text {Classic-DM }}, 25 \%$ were equivalent to $C E B P A^{\mathrm{WT}}$, and $44 \%$ were intermediate between the two. Similar heterogeneity has been reported for gene expression profiles of non-classic cases, with 3 of 7 such cases segregating from cases with classical mutants. ${ }^{20}$ This variability is not surprising considering the diversity of the mutant combinations. Although the p30 isoform is thought to play an important role in allowing commitment of the leukemic stem cell to the myeloid lineage, ${ }^{38}$ the mechanism by which it promotes AML is not

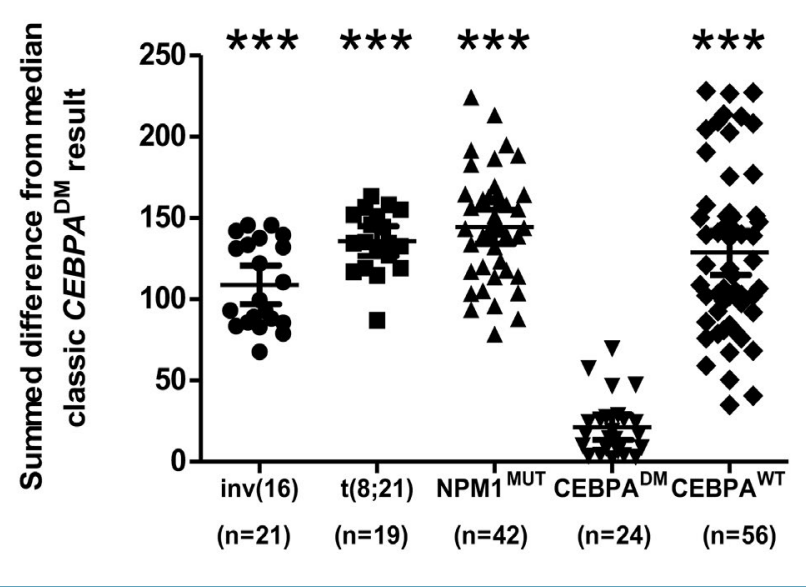

Figure 5. Methylation levels in other good-risk groups compared to the CEBPA ${ }^{\text {Classic-DM }}$ cases. A composite methylation score was calculated by summing the difference between the \% methylation for samples and the median for the 24 CEBPA ${ }^{\text {classic-DM }}$ cases (excluding the outlier) for three differentially methylated CpG sites: KHNYN, VAMP5 and LY9. Mean values $\pm 95 \%$ confidence intervals are shown. The CEBPA $A^{\text {classic-DM }}$ and CEBPA ${ }^{\text {wT }}$ results were $\beta$ values from the arrays: results for the three comparative groups were obtained by pyrosequencing. Significance refers to difference from the CEBPA ${ }^{\text {Classi-DM }}$ group. $* * * P \leq 0.001$.

clearly defined. It has a lower affinity for some C/EBP sites than p42 and induces multiple genes that are not affected by $p 42,39-41$ and this may have influenced the methylation profile. Knock/in mice expressing just $\mathrm{N}$-terminal mutant developed leukemia but more slowly than the $\mathrm{N}+\mathrm{C}$ combination. ${ }^{38,42}$ This presumably reflects the additional influence of an aberrant C-terminally mutated protein that might not bind to DNA but can still bind to other C/EBP interacting proteins, such as PU.1 and the SWI/SNF com- 
plex. Classic C-terminal mutants are associated with hyperproliferation due to loss of cell-cycle regulation and a block in myeloid differentiation. ${ }^{43}$ Although knock/in mice with classic $\mathrm{C}$ mutant alone do develop leukemia, it is with slower latency than the $\mathrm{N}+\mathrm{C}$ and mutant $\mathrm{N}$ mice. ${ }^{42}$ Since these mutants may still bind and potentially sequester other interacting factors, it has been suggested that this could limit the ability of other C/EBPs to rescue the effect of the aberrant $\mathrm{C} / \mathrm{EBP} \alpha,{ }^{2}$ as shown for $\mathrm{C} / \mathrm{EBP} \beta$ in the $\mathrm{C} / \mathrm{EBP} \alpha$-deficient situation. ${ }^{44,45}$ This more global cellular impact of the C-terminal mutants may have a greater consequence for signaling events downstream of $\mathrm{C} / \mathrm{EBP} \alpha$, which may, therefore, be reflected in the methylation profile.

The methylation profiles did not group according to the predicted functional consequence of the mutant, whether $\mathrm{N}$ - or C-terminally mutated. For example, considerable variability was observed for the cases with a C-missense mutation. Of the three classic $\mathrm{N}$ plus C-missense combinations assayed, 1 case had a methylation profile equivalent to CEBPA ${ }^{\mathrm{WT}}$ and 2 cases had intermediate profiles. Two homozygous C-missense cases grouped with $C E B P A^{\mathrm{WT}}$ cases, whereas another homozygous C-missense case grouped with the CEBPA ${ }^{\text {Classic-DM }}$ cases. In the 51 cases documented with C-missense mutations in the COSMIC database (http://cancer.sanger.ac.uk/cosmic), most mutations are unique, reported in $1(n=27,53 \%)$ or $2(n=9$, $18 \%$ ) patients, and only two residues (R297 and R300) are recorded as being variably mutated in 5 patients. Critical amino acids at the bZIP/DNA interface have been identified from the C/EBP $\alpha$ crystal structure, but many additional hydrogen bonds and van der Waals contacts are implicated in the stabilization of these interactions. ${ }^{46}$ Thus, predicting the functional consequence of these mutants is difficult.

From a clinical perspective, risk management requires evaluation of all the available information, and the data presented here suggest that CEBPA ${ }^{\mathrm{DM}}$ patients with a nonclassic mutation should not automatically be included in the same favorable-risk prognostic group as CEBPA Classic-DM cases; it might be appropriate to consider them for allogeneic transplantation in first remission. Ultimately, this can only be proven by analysis of clinical trial outcomes and, with the increasing availability of large data sets using targeted next-generation sequencing panels, such analysis may be feasible in the future. This will also promote a better understanding of the mutational background of classic and non-classic CEBPA ${ }^{\mathrm{DM}}$ cases and whether there are differences that impact on their prognosis. Our studies also raise the possibility that methylation profiling may identify those non-classic cases that behave in a similar manner to classic mutants; although we can-
A

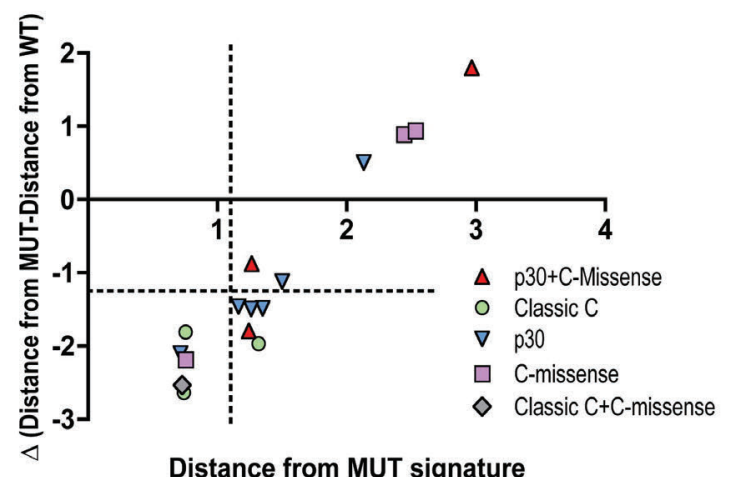

B

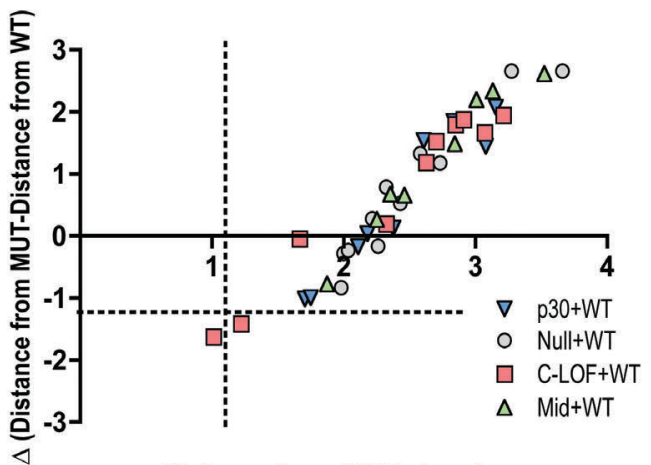

Distance from MUT signature

Figure 6. Supervised analysis of $C E B P A^{\mathrm{DM}}$ cases with non-classic mutations and

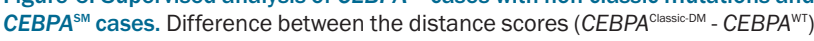
compared with the distance from the CEBPA ${ }^{\text {classic:DM }}$ (MUT) signature for $(A)$ $C E B P A^{\mathrm{DM}}$ cases with a non-classic mutant. (B) $C E B P A^{\mathrm{SM}}$ cases.

not directly attribute a causal link between the methylation pattern and chemosensitivity, and further studies are required before this is introduced into clinical practice.

\section{Acknowledgments}

The authors would like to thank Kerra Pearce at UCL Genomics for technical assistance.

\section{Funding}

This work was supported by Leukaemia and Lymphoma Research, now called Bloodwise, the UK Medical Research Council and Cancer Research UK, and was undertaken at UCL, which receives a proportion of funding from the Department of Health's NIHR Biomedical Research Centre's funding scheme.

\section{References}

1. Friedman $\mathrm{AD}$. C/EBPalpha in normal and malignant myelopoiesis. Int $\mathrm{J}$ Hematol. 2015;101(4):330-341.

2. Ohlsson E, Schuster MB, Hasemann M, Porse BT. The multifaceted functions of $\mathrm{C} / \mathrm{EBP}$ alpha in normal and malignant haematopoiesis. Leukemia. 2016;30(4):767775 .
3. Wouters BJ, Lowenberg B, ErpelinckVerschueren CA, van Putten WL, Valk PJ, Delwel R. Double CEBPA mutations, but not single CEBPA mutations, define a subgroup of acute myeloid leukemia with a distinctive gene expression profile that is uniquely associated with a favorable outcome. Blood. 2009;113(13):3088-3091.

4. Dufour A, Schneider F, Metzeler KH, et al. Acute myeloid leukemia with biallelic CEBPA gene mutations and normal kary- otype represents a distinct genetic entity associated with a favorable clinical outcome. J Clin Oncol. 2010;28(4):570-577

5. Pabst T, Eyholzer M, Fos J, Mueller BU Heterogeneity within AML with CEBPA mutations; only CEBPA double mutations, but not single CEBPA mutations are associated with favourable prognosis. $\mathrm{Br} \mathrm{J}$ Cancer. 2009;100(8):1343-1346.

6. Green CL, Koo KK, Hills RK, Burnett AK, Linch DC, Gale RE. Prognostic significance 
of CEBPA mutations in a large cohort of younger adult patients with acute myeloid leukemia: impact of double CEBPA mutations and the interaction with FLT3 and NPM1 mutations. J Clin Oncol. 2010; 28(16):2739-2747.

7. Taskesen E, Bullinger L, Corbacioglu A, et al. Prognostic impact, concurrent genetic mutations, and gene expression features of AML with CEBPA mutations in a cohort of 1182 cytogenetically normal AML patients: further evidence for CEBPA double mutant AML as a distinctive disease entity. Blood. 2011;117(8):2469-2475.

8. Fasan A, Haferlach C, Alpermann T, et al. The role of different genetic subtypes of CEBPA mutated AML. Leukemia. 2014; 28(4):794-803.

9. Li HY, Deng DH, Huang Y, et al. Favorable prognosis of biallelic CEBPA gene mutations in acute myeloid leukemia patients: a meta-analysis. Eur J Haematol. 2015; 94(5):439-448.

10. Dohner H, Estey EH, Amadori S, et al. Diagnosis and management of acute myeloid leukemia in adults: recommendations from an international expert panel, on behalf of the European LeukemiaNet. Blood. 2010;115(3):453-474

11. O'Donnell MR, Abboud CN, Altman J, et al. Acute myeloid leukemia. J Natl Comp Cancer Network. 2012;10(8):984-1021.

12. Ofran Y, Rowe JM. Genetic profiling in acute myeloid leukaemia--where are we and what is its role in patient management. Br J Haematol. 2013;160(3):303-320.

13. Cornelissen JJ, Gratwohl A, Schlenk RF, et al. The European LeukemiaNet AML Working Party consensus statement on allogeneic HSCT for patients with AML in remission: an integrated-risk adapted approach. Nat Rev Clin Oncol. 2012; 9(10):579-590.

14. Nerlov C. C/EBPalpha mutations in acute myeloid leukaemias. Nat Rev Cancer. 2004:4(5):394-400

15. Fitzgibbon J, Smith LL, Raghavan M, et al. Association between acquired uniparental disomy and homozygous gene mutation in acute myeloid leukemias. Cancer Res. 2005;65(20):9152-9154

16. Wouters BJ, Sanders MA, Lugthart $S$, et al. Segmental uniparental disomy as a recurrent mechanism for homozygous CEBPA mutations in acute myeloid leukemia. Leukemia. 2007;21(11):2382-2384.

17. Shih LY, Liang DC, Huang CF, et al. AML patients with CEBPalpha mutations mostly retain identical mutant patterns but frequently change in allelic distribution at relapse: a comparative analysis on paired diagnosis and relapse samples. Leukemia. 2006;20(4):604-609.

18. Benthaus T, Schneider F, Mellert G, et al. Rapid and sensitive screening for CEBPA mutations in acute myeloid leukaemia. Br J Haematol. 2008;143(2):230-239.

19. Behdad A, Weigelin HC, Elenitoba-Johnson KS, Betz BL. A clinical grade sequencingbased assay for CEBPA mutation testing: report of a large series of myeloid neo- plasms. J Mol Diag. 2015:17(1):76-84.

20. Lavallee VP, Krosl J, Lemieux S, et al. Chemo-genomic interrogation of CEBPA mutated AML reveals recurrent CSF3R mutations and subgroup sensitivity to JAK inhibitors. Blood. 2016;127(24):3054-3061.

21. Wouters BJ, Jorda MA, Keeshan K, et al Distinct gene expression profiles of acute myeloid/T-lymphoid leukemia with silenced CEBPA and mutations in NOTCH1. Blood. 2007;110(10):3706-3714.

22. Figueroa ME, Lugthart S, Li Y, et al. DNA methylation signatures identify biologically distinct subtypes in acute myeloid leukemia. Cancer Cell. 2010;17(1):13-27.

23. Cancer Genome Atlas Research Network, Ley TJ, Miller C, et al. Genomic and epigenomic landscapes of adult de novo acute myeloid leukemia. New Engl J Med. 2013;368(22):2059-2074

24. Figueroa ME, Wouters BJ, Skrabanek L, et al. Genome-wide epigenetic analysis delineates a biologically distinct immature acute leukemia with myeloid/T-lymphoid features. Blood. 2009:113(12):2795-2804.

25. Sproul D, Kitchen RR, Nestor CE, et al. Tissue of origin determines cancer-associated CpG island promoter hypermethylation patterns. Genome Biol. 2012;13(10):R84.

26. Sproul D, Nestor C, Culley J, et al. Transcriptionally repressed genes become aberrantly methylated and distinguish tumors of different lineages in breast cancer. Proc Natl Acad Sci USA. 2011; 108(11):4364-4369.

27. Illingworth RS, Gruenewald-Schneider U, Webb S, et al. Orphan CpG islands identify numerous conserved promoters in the mammalian genome. PLoS Gen. 2010; 6(9):e1001134.

28. Gale RE, Lamb K, Allen C, El-Sharkawi D, Stowe C, Jenkinson S, et al. Simpson's Paradox and the Impact of Different DNMT3A Mutations on Outcome in Younger Adults With Acute Myeloid Leukemia. J Clin Oncol. 2015;33(18):20722083.

29. Pabst T, Mueller BU, Harakawa N, et al. AML1-ETO downregulates the granulocytic differentiation factor C/EBPalpha in $\mathrm{t}(8 ; 21)$ myeloid leukemia. Nat Med. 2001; 7(4):444-451.

30. Ptasinska A, Assi SA, Martinez-Soria N, et al. Identification of a dynamic core transcriptional network in $t(8 ; 21)$ AML that regulates differentiation block and selfrenewal. Cell Rep. 2014;8(6):1974-1988.

31. Helbling D, Mueller BU, Timchenko NA, et al. CBFB-SMMHC is correlated with increased calreticulin expression and suppresses the granulocytic differentiation factor CEBPA in AML with inv(16). Blood. 2005;106(4):1369-1375

32. Papaemmanuil E, Gerstung M, Bullinger L, et al. Genomic Classification and Prognosis in Acute Myeloid Leukemia. New Engl Med. 2016:374(23):2209-2221.

33. Grossmann V, Haferlach C, Nadarajah N, et al. CEBPA double-mutated acute myeloid leukaemia harbours concomitant molecular mutations in $76.8 \%$ of cases with TET2 and GATA2 alterations impacting prognosis. Br J Haematol. 2013;161(5):649-658.

34. Green CL, Tawana K, Hills RK, et al GATA2 mutations in sporadic and familial acute myeloid leukaemia patients with CEBPA mutations. Br J Haematol. 2013;161(5):701-705.

35. Theis F, Corbacioglu A, Gaidzik VI, et al. Clinical impact of GATA2 mutations in acute myeloid leukemia patients harboring CEBPA mutations: a study of the AML study group. Leukemia. 2016;30(11):2248-2250.

36. Heath V, Suh HC, Holman M, et al. C/EBPalpha deficiency results in hyperproliferation of hematopoietic progenitor cells and disrupts macrophage development in vitro and in vivo. Blood. 2004;104(6):16391647.

37. Zhang P, Iwasaki-Arai J, Iwasaki $\mathrm{H}$, et al Enhancement of hematopoietic stem cell repopulating capacity and self-renewal in the absence of the transcription factor C/EBP alpha. Immunity. 2004;21(6):853863.

38. Kirstetter P, Schuster MB, Bereshchenko O, et al. Modeling of C/EBPalpha mutant acute myeloid leukemia reveals a common expression signature of committed myeloid leukemia-initiating cells. Cancer Cell. 2008;13(4):299-310.

39. Cleaves $R$, Wang $O F$, Friedman $A D$. C/EBPalphap30, a myeloid leukemia oncoprotein, limits G-CSF receptor expression but not terminal granulopoiesis via siteselective inhibition of C/EBP DNA binding. Oncogene. 2004;23(3):716-725.

40. Geletu M, Balkhi MY, Peer Zada AA, et al. Target proteins of C/EBPalphap30 in AML: C/EBPalphap30 enhances sumoylation of C/EBPalphap42 via up-regulation of Ubc9. Blood. 2007;110(9):3301-3309.

41. Wang C, Chen X, Wang Y, Gong J, Hu G. C/EBPalphap30 plays transcriptional regulatory roles distinct from C/EBPalphap42. Cell Res. 2007;17(4):374-383

42. Bereshchenko O, Mancini E, Moore S, et al Hematopoietic stem cell expansion precedes the generation of committed myeloid leukemia-initiating cells in C/EBPalpha mutant AML. Cancer Cell. 2009;16(5):390400.

43. Kato N, Kitaura J, Doki N, et al. Two types of C/EBPalpha mutations play distinct but collaborative roles in leukemogenesis: lessons from clinical data and BMT models. Blood. 2011;117(1):221-233.

44. Jones LC, Lin ML, Chen SS, et al Expression of C/EBPbeta from the C/ebpalpha gene locus is sufficient for normal hematopoiesis in vivo. Blood. 2002; 99(6):2032-2036.

45. Hirai $\mathrm{H}$, Zhang $\mathrm{P}$, Dayaram $\mathrm{T}$, et al C/EBPbeta is required for 'emergency' granulopoiesis. Nat Immunol. 2006; 7(7):732-739

46. Miller M, Shuman JD, Sebastian T, Dauter Z, Johnson PF. Structural basis for DNA recognition by the basic region leucine zipper transcription factor CCAAT/enhancerbinding protein alpha. J Biol Chem. 2003; 278(17):15178-15184. 